\title{
DE LOVECRAFT A ALEMÁN: UNA ADAPTACIÓN CINEMATOGRÁFICA DE LOS MITOS DE CTHLHU
}

\section{FROM LOVECRAFT TO ALEMÁN: \\ A CINEMATOGRAPHIC ADAPTATION OF THE CTHULHU MYTHOS}

Mikel Peregrina Castaños

Universidad Complutense de Madrid, España peretorian@gmail.com

\section{Resumen:}

La influencia de H. P. Lovecraft y de los Mitos de Cthulhu ha trascendido de la propia literatura a otros ámbitos culturales, como el cine. En concreto, los intentos de adaptación no han resultado muy acertados, ante la dificultad de lidiar con una prosa excesivamente descriptiva, de ritmo lento y con escasez de personajes. En el cine español, este reto ha sido asumido recientemente por José Luis Alemán en un díptico: La herencia Valdemar y La sombra prohibida. Un análisis de ambos filmes arrojará luz sobre el valor del proyecto de Alemán, indicará cuál es su deuda con el escritor de Providence, y destacará aquellos puntos en los que se aleja de modelo literario.

\begin{abstract}
:
The influence of H. P. Lovecraft and the Cthulhu Mythos has gone beyond the literature to other cultural fields, like the cinema. In particular, the attempts of film adaptations haven't turned out really correct, faced with the difficulty to deal with a thoroughly descriptive prose, of slow rhythm and with a lack of characters. In the Spanish cinema, this challenge has been accepted by José Luis Alemán with a film diptych: La herencia Valdemar and La sombra prohibida. An analysis of both films will shed light on the value of Aleman's project, will indicate what his debt with the author from Providence is, and will underline those points in which he gets away from the literary model.
\end{abstract}

Palabras claves: cine español; cine de terror; H. P. Lovecraft; José Luis Alemán; La herencia Valdemar.

Keywords: Spanish Cinema; Horror Cinema; H. P. Lovecraft; José Luis Alemán; La herencia Valdemar. 


\section{La influencia de Lovecraft y de los Mitos de Ctulhu}

En vida Howard Phillips Lovecraft (1890-1937) nunca tuvo en alta estima su obra, publicada en general en revistas populares, como Weird Tales. Sin embargo, sus escritos fantásticos han ido adquiriendo relevancia hasta otorgarle la fama de la que goza en la actualidad. Este renombre se debe a la labor difusora de admiradores como August Derleth y Donald Wandrei, fundadores de la editorial Arkham House.

Hoy en día, H. P. Lovecraft es recordado, dentro de la historia de la literatura de terror, gracias al ciclo de relatos que August Derleth y otros organizaron como si fuera una mitología, los Mitos de Cthulhu. Esta es una de las cosmogonías más influyentes de la literatura fantástica donde los Primordiales o Primigenios invaden nuestra realidad desde una suprarrealidad, desmoronando las leyes de la naturaleza que creíamos inmutables. El ciclo está conformado por poco más de una docena de escritos cuyo atractivo reside en constituir un mundo ficcional indefinido, impreciso, sólo sugerido, donde las piezas dejan interrogantes en el aire, donde el autor evita precisar la distribución de fuerzas y poderes, y en el cual libros y cultos que los alaban lo hacen en términos confusos y contradictorios.

El éxito de los Mitos de Cthulhu ha absorbido la figura del propio escritor, que también ha sido mitificada, dada su imagen de devorador de libros sobre la antigüedad y demiurgo de horrores sobrenaturales (Hernández de la Fuente, 2005, p. 23). Como consecuencia, siguiendo a Michel Houellebecq, "la figura del recluso de Providence se ha convertido en algo tan mítico como sus propias creaciones" (Houellebecq, 1991, p. 30), suscitando la admiración e imaginación de las generaciones venideras.

La particularidad de esta mitología reside en que es un sistema abierto, sugerente, cuyos vacíos invitaron a nuevas aportaciones originales. Como consecuencia, citando a David Hernández de la Fuente, "Lovecraft, en cierto modo, estableció los fundamentos de un nuevo paganismo literario que ha tenido numerosos acólitos después de su muerte" (2005, p. 21). Acorde con la visión de Rafael Llopis, "los Mitos de Cthulhu son, pues, obra colectiva que cristalizó en torno a un hombre solitario" (1969, p. 22), lo que ha transformado esta mitología en un universo compartido. Y dicha colectividad empezó con los 
amigos del escritor de Providence, que conformaron el llamado Círculo de Lovecraft, un grupo de escritores cuyo contacto con el escritor de Providence suscitó una motivación por contribuir al desarrollo literario de la difusa idea inicial de Lovecraft (Muñoz Casado, 2012, p. 242). Más relevante aún es que su influencia trasciende de la literatura a otros ámbitos artísticos, lo que supone un destacado trasvase cultural hasta crear todo un culto popular.

La inmensa mayoría de creadores y autores, ya sean escritores, cineastas, diseñadores, pintores o escultores, que han trabajado temas y conceptos relacionados con el terror y el miedo se han enfrentado tarde o temprano con la obra de Lovecraft. No es de extrañar por tanto que muchos de ellos hayan querido homenajearle de manera voluntaria o bien no hayan podido evitar el influjo de aquél en sus propias creaciones (Arenas Orient, 2011, p. 241).

Todo ello ha conformado una extensa cultura popular en torno a la obra de Lovecraft. De ese modo, encontramos referencias a Lovecraft y/o a los Mitos de Cthulhu en la música, con canciones como "The Call of Kthulhu” -en el álbum Ride the Lightning (1984)-, de Metallica; en el cómic, como Necronomicon (2010), escrito por Alan Moore e ilustrado por Jacen Burrows; en videojuegos, como Alone in the dark (1982), desarrollado por Infogrames; en juegos de mesa, como Arkham Horror (1987), diseñado por Richard Launius; y, claramente, en el cine, el ámbito concreto que aquí nos ocupa. Sin embargo, en esta popularización la esencia lovecraftiana se ha ido desvirtuando, con la consiguiente dificultad de acceder a la pureza del sentido de sus textos, cuanto mayor es el carácter mediático de esos medios y más se alejan de un carácter cultural minoritario o marginal (Jones, 2013, p. 230).

La problemática de estas películas basadas en Lovecraft reside en la dificultad de adaptación del texto literario. Ello constituye un reto para cualquier director que decida adaptar a H. P. Lovecraft. En el cine español, ese reto ha sido asumido recientemente por el director novel José Luis Alemán en un díptico fílmico: La herencia Valdemar (2010a) y La sombra prohibida (2011). Como consecuencia, analizar las similitudes y discrepancias de estas películas con respecto a los escritos de Lovecraft ayudará a desentrañar cuál es la parte de la obra del maestro de Providence que ha sobrevivido en este trasvase cultural concreto. 


\section{Lovecraft en el cine: las dificultades de adaptación de su obra}

Los textos de Lovecraft empezaron a adaptarse al séptimo arte en los años sesenta. A ello influyó, en parte, el éxito de las adaptaciones que Roger Corman empezó a realizar sobre diferentes obras de Poe (Smith, 2005, p. 119), en las cuales este director sintetizaba lo mejor de las películas de la productora Hammer con la renovación del horror creada por Hitchcock con Psicosis (Psycho, 1960). Sin embargo, los intentos de adaptar la obra de Lovecraft al cine no han obtenido notable acierto y normalmente han sido catalogados como cine de serie B. En términos generales, partiendo de la premisa de David Hernández de la Fuente, se trata de "un cine que poco tiene que ver con Lovecraft: ya lejos del críptico mundo de H. P. Lovecraft, vierte los elementos más básicos de sus mitos en la olla de la cultura de masas" (2005, p. 77).

Tal y como se aclarará más adelante, más allá del concepto de horror cósmico propugnado por el propio Lovecraft, estas adaptaciones han huido del carácter sugestivo del escritor de Providence para incurrir en diferentes procesos de vulgarización inexistentes en los textos originales. De este modo, algunas de ellas se han centrado en destacar la monstruosidad, por ejemplo, en El innombrable (The Unnamable, 1988), de Jean-Paul Ouellette. En otras ocasiones, como sucede en Re-Animator (1985), de Stuart Gordon, han mezclado la solemnidad y seriedad lovecraftiana con toques humorísticos. Y en otros ejemplos se han preocupado más por desarrollar lo visceral, lo gore, donde lo extraordinario y sobrenatural es accesorio para mostrar más elementos que provoquen repugnancia (García Mingorance, 2014, p. 222). Es el caso de Infinitamente Endemoniado (Lurking Fear, 1994), de C. Courtney Joyner.

Las excepciones más notables en este punto probablemente sean el mediometraje de Andrew Leman, The Call of Cthulhu (2005) y The Whisperer in Darkness (2011), de Sean Branney. Ambas producciones fueron respaldadas por la H. P. Lovecraft Historical Society, lo cual influye en la fidelidad y el respeto por los textos originales. Pero, a la vez, constituyen nuevos productos cultural mediante los recursos propios de la cinematografía. Los directores consiguen evocar el espíritu lovecraftiano gracias a una cuidada puesta en escena y una ambientación contemporánea al escritor mediante el uso del blanco y negro. Esta fidelidad es todavía más determinante en el caso de 
Lewman, puesto que se retrotrajo a la tecnología de la época para realizar The Call of Cthulhu mediante la inclusión de intertítulos propios del cine mudo, e incluso el stop motion para recrear a Cthulhu. Siguiendo a Jesús Palacios en su crítica a este mediometraje concreto:

Todo en The Call of Cthulhu, hasta los más pequeños detalles (las estatuillas y los dibujos, los libros sobre la Atlántida que leen los personajes, los recortes de prensa, etc.), evoca e invoca con conocimiento el mundo arcano, teosófico, esotérico y pulp del Maestro de Providence (2008, pp. 198-199).

A diferencia de las adaptaciones cinematográficas de textos de Lovecraft, las adaptaciones indirectas del terror lovecraftiano sí han conseguido una repercusión mayor dentro de la historia del cine ${ }^{1}$. Si, entonces, los principales éxitos cinematográficos de la obra lovecraftiana son influencias indirectas y no adaptaciones directas, es necesario preguntarse a qué se debe este hecho.

A pesar de las múltiples adaptaciones, el estilo de Lovecraft es poco propicio para su trasvase al ámbito cinematográfico. En palabras de Don G. Smith, "Lovecraft's more philosophically challenging and chilling viewpoints have rarely been adequately translated to the screen" (2005, p. 119). Muchos son los críticos que han calificado su escritura de barroca y encorsetada, donde abundan las descripciones y los análisis o pensamientos del protagonista, lo que ralentiza considerablemente el ritmo narrativo y reduce la acción del relato ${ }^{2}$. Tal y como alude David Hernández de la Fuente:

Las descripciones de Lovecraft, en el clímax del relato, son barrocas, arcaicas, demenciales: un torbellino de palabras que aturde cada sentido del lector y ataca la base de sus convicciones [...]. Es la estética de lo abominable, el ansia de provocar el horror a través del barroquismo, de la obsesiva acumulación hacia el clímax de epítetos (Hernández de la Fuente, 2005, pp. 54-55).

Ello es fruto de la dialéctica entre el materialismo científico y la necesidad de trascendencia sobre la realidad que se percibe como tónica general en su obra. Si algo le caracteriza es la introducción del cientifismo con la idea de diseccionar con escalpelo lo innombrable, de ahí la profusión de adjetivos. En palabras de

\footnotetext{
1. Es el caso de Alien (1979), de Ridley Scott, y The Thing (1982), de John Carpenter. La relación de ambas películas con el escritor de Providence ya ha sido indicada en diversas ocasiones. Véase a este respecto el artículo de Carlos Arenas Orient (2011).

2. Me remito aquí a muchas de las opiniones que aparecen en el documental Lovecraft: Fear of the Unknown (2008), de Frank H. Woodward.
} 
Fernando Darío González Grueso, en Lovecraft "se vierte realismo en las descripciones para crear un ambiente propicio para el evento sobrenatural, presentado desde el raciocinio científico, y más allá de los acontecimientos terrenos, el horror de influencia cosmogónica atrapa a los personajes en un círculo sin salida” (González Grueso, 2013, pp. 96-97).

Por su parte, siguiendo a Teodoro Gómez, podemos destacar los siguientes rasgos generales: a) el abuso de adjetivos, recurso básico para crear la atmósfera; b) el inglés arcaico, pretensión de Lovecraft porque sus narraciones estuvieran escritas por los mismos eruditos que las protagonizaban; c) la primera persona, para la reafirmación del propio yo del escritor y como caja de resonancia para los acontecimientos brutales y sorprendentes que generalmente le suceden a otro; d) la ausencia de personajes femeninos y de alusiones al sexo y al dinero (Gómez, 2002, pp. 74 y ss.).

Hay en sus escritos una ausencia casi total de diálogos, desarrollándose el relato a través de esa narración homodiegética intradiegética con focalización interna3, un modelo de voz narradora tan caracterizador de la literatura contemporánea y que parte del relativismo del personaje. De ese modo, crea un tipo de horror muy introspectivo, que aboga por sugerir más que por mostrar, manteniendo el misterio incluso una vez acabado el relato. Además, se decanta por la elección de personajes masculinos, a los cuales a menudo sitúa al borde de la locura o el suicidio, producto de haberse cruzado por su vida el hecho sobrenatural.

Si se compara con el lenguaje cinematográfico, sostenido en el poder de la imagen, se comprueba la distancia entre ambas técnicas narrativas. Entre otros aspectos, el cine opta hacia una focalización externa, la que muestra la cámara. Así, en palabras de Josep María Catalán, en el cine "la relatividad del punto de vista se produce en la supuesta objetividad de una escena: se combinan, al parecer, los potenciales de la novela realista en tercera persona con los de la novela relativista con varias perspectivas" (2001, p. 149). Con ello la cámara provoca un efecto de distanciamiento y, a la vez, de identificación con la parte enfocada, lo que complica la introspección presente en los textos del escritor de Providence. Además, la imagen es simultanea -llega en presente-, y no deja espacio a las reflexiones propias del protagonista para crear el horror

3 Para estos términos de narratología, véase G. Genette, Figuras III (1972). 
introspectivo lovecraftiano. Ese predominio de la acción interior -psicológicadel protagonista en Lovecraft dificulta, por tanto, el trasvase al ámbito cinematográfico 4 .

A ello deben sumarse dos argumentos clave que explican el fracaso de muchas adaptaciones de la obra de H. P. Lovecraft. Por un lado, dada la finalidad mercantilista de una enorme porción la producción cinematográfica, los guionistas generalmente se dirigen a audiencias masivas, por lo que su trabajo no puede ser excesivamente intelectual, justamente lo contrario a lo que buscaba Lovecraft con su estilo. Por otro lado, la mayor parte del cine de terror se dirige a una audiencia de adolescentes, lo que requiere que el ritmo sea veloz, la acción trepidante y mantenga al público entretenido. Como consecuencia, la idea de Lovecraft de que el humanocentrismo es absurdo y de que la humanidad está sola en el abismo no resulta un mensaje tranquilizador para la masa de espectadores y además constituye un mensaje que mueve a la reflexión en el espectador, y no al mero entretenimiento. En palabras de Christopher Sharrett, "his black vision and sly humor are totally lost, however, on most of the horror filmmakers whose works would not exist without him” (2015, p. 23).

Por tanto, según se ha indicado previamente, los distintos directores que se han topado con la dificultad de lidiar con el estilo lovecraftiano no han sido en general capaces de solventar este escollo a la vez que procuraban mantenerse fieles a la obra del escritor de Providence. Siguiendo a Don G. Smith, casi ningún filme basado en Lovecraft "has adequately captured the author's materialistic sense of the universe and his view of humanity's smallness and insignificance in the fate of a vast, indifferent universe" (2005, p. 120).

\section{La apuesta de José Luis Alemán: La herencia Valdemar y La sombra prohibida}

Como ya se ha indicado, en el cine español, el reto de adaptar a Lovecraft fue asumido recientemente por José Luis Alemán con dos películas: La herencia Valdemar (2010a) y La sombra prohibida (2011). Inicialmente, hay que destacar que no es el primer caso de adaptación lovecraftiana que aparece en el

4 Para un mayor entendimiento de las diferencias entre el punto de vista narrativo y el cinematográfico, véase Catalán (2001, pp. 129-165). 
cine en nuestro país, puesto que con anterioridad ya había participado España en la producción de Dagon, la secta del mar (Dagon, 2001)5, de Stuart Gordon, y Beyond Re-Animator (2003), de Brian Yuzna. Se trata, realmente, de películas insertas en la estela de la productora Fantastic Factory, la empresa española creada por Brian Yuzna y Julio Fernández y que pervivió desde 2001 hasta 2007.

A diferencia de los casos anteriores, el proyecto de Alemán se erige sobre otros parámetros. En primer lugar, hay que señalar que no estamos ante una adaptación cinematográfica de un texto lovecraftiano concreto (quizás el mayor parentesco lo pueda mantener la primera parte con The Dunwich Horror), sino que la coincidencia se produce entre mundos ficcionales, puesto que la trama del díptico de Alemán se inserta dentro de los Mitos de Cthulhu. Ello conlleva que el trasvase cultural dé lugar a una obra nueva, repleta de elementos propios del universo ideado por Lovecraft, eliminando con ello un posible carácter subsidiario respecto a un escrito literario que sirviera de base, algo que sucede en muchas de las adaptaciones anteriormente citadas.

Precisamente, sobre la obra de H. P. Lovecraft, señaló José Luis Alemán en la rueda de prensa previa al estreno de La herencia Valdemar que se trata de un escritor "muy descriptivo, muy denso y complicado. La idea ha sido coger la esencia de su obra y adaptarla a un guión libre, adornado con elementos históricos que aporten credibilidad y riqueza a la producción” (en Arce, 2010, Web).

En ese sentido, hablamos de un trasvase parcial, donde la influencia de la cultura popular en los Mitos de Cthulhu es muy marcada. Tomando la terminología de José Luis Sánchez Noriega (2000, pp. 63-72), se trata, pues, de una adaptación libre. José Luis Alemán toma un universo compartido para realizar una historia propia donde incluye otro tipo de fuentes a parte de la

\footnotetext{
5. Sobre esta producción en concreto, señalan Diego López y David Pizarro que en el filme "escenarios decadentes, sacrificios y atmósferas aceptables nos conducen hasta un final intimista, revelador de antiguos secretos familiares, donde el desconcierto y la anarquía, algo imperante en todo el metraje, vuelve a estar acompañado de un reparto errático" (2013: 384). Por su parte, Carlos Aguilar indica que "Dagon. La secta del mar convence por continuos aciertos de tono y atmósfera, aunque fracasa en su propósito de reunir a los amantes del Fantastique literario, por lo común de una cierta edad, y los amantes del Gore, en general jóvenes reacios a la lectura", y añade más adelante que dicha película llegó a ser "un film español de horror fantástico competitivo a escala internacional y nada indigno de su inspiración literaria" (2005, p. 39).
} 
mitología lovecraftiana. Como consecuencia, se produce una reelaboración que en este caso toma un mundo ficcional como esquema argumental, por lo que se recrea un mito literario que forma parte de un imaginario colectivo.

Continuando con la clasificación de Sánchez Noriega, según el tipo de relato, hablaríamos de divergencia estilística, puesto que actualiza en diversos puntos los textos de H. P. Lovecraft. Según la extensión, hablamos de una ampliación, ya que, a pesar de que Alemán no se basa en un texto lovecraftiano específico, sí incluye muchos otros elementos en la trama cuya presencia responde a las otras influencias del díptico cinematográfico español. Finalmente, según la propuesta estético cultural, se incurre en algunos procesos de vulgarización, como la actualización, que introduce en las obras clásicas sensibilidades contemporáneas y diseña personajes y dramatizaciones que intentan acercarse al gusto actual. En palabras del propio José Luis Alemán en una nota en la Web de La sombra prohibida:

Tuvimos la suerte de ir introduciéndonos en el mundo de H. P. Lovecraft y descubrir que dentro del cine fantástico en torno a su obra había mucho que desarrollar aún; daba la sensación de que era un conjunto de ideas que se habían ido quedando en un cajón y nadie las había tocado nunca. Pero nos daba miedo introducirnos en toda su literatura, por lo que decidimos extraer la esencia de su universo y adaptarlo a un guión propio, para intentar llegar a un público que no estuviera iniciado en la literatura de H. P. Lovecraft (Alemán 26/9/2016: Web).

Alemán busca con ello su propia autonomía estética dentro, incluso, de la tradición de la cinematografía relacionada con la obra de Lovecraft. El motivo que mueve la adaptación de Alemán, siguiendo los presupuestos de Sánchez Noriega (2000, pp. 50-56), sería en parte la de labor divulgadora, en parte la necesidad de historias y en parte la pretensión de recrear de forma personal un mito literario. Tanto La herencia Valdemar como La sombra prohibida -que en principio iban a constituir una trilogía, pero que se redujo por razones de presupuesto- bebe, además de la obra de H. P. Lovecraft, como se ha indicado, de las fuentes del folletín decimonónico y del cine de terror clásico. Esta mezcla de fuentes reduce el peso que los elementos lovecraftianos acabarán teniendo en el díptico de Alemán. 
Por ello, constituye una monster movie que, según declaraciones de José Luis Alemán en la entrevista que le hizo Fernández Pinilla, rehúye del gore y del susto fácil que tanto parece dominar en el cine de terror más reciente. Como consecuencia, ellos se propusieron retornar hacia un terror clásico, romántico (Fernández Pinilla, 2008). De ahí el punto de originalidad de la producción española, dado el riesgo que supone desligarse del vigente gusto del espectador en un cine de género para recuperar el de una época anterior.

A modo de sinopsis, La herencia Valdemar y su continuación La sombra prohibida, se sustentan sobre los modelos de la casa embrujada y de los ritos ocultos típicos de los relatos de Lovecraft. Al comienzo, Luisa Llorente (Silvia Abascal), tasadora de fincas, acude a valorar la mansión Valdemar, situada en una alejada e incomunicada población, Montenegro, pero desaparece sin dejar rastro. La compañía, Inmoberance, envía, por un lado, al detective Nicolás Trámel (Óscar Jaenada), y por otro, a Ana (Norma Ruiz) y Eduardo Campins (Rodolfo Sancho), compañeros de Luisa, que viajan en coche con la idea de poder unir sus fuerzas con el investigador privado.

Una vez allí todos se descubren como víctimas de un complot perpetrado por el jefe de la compañía, Maximilian Colvin (Eusebio Poncela), a su vez líder de una secreta secta adoradora de los Primigenios, que se propone sacrificarlos para invocar al poderoso Cthulhu. $\mathrm{Al}$ rito acude también el imposiblemente nada avejentado Lázaro Valdemar (Daniele Liotti), el dueño de la casa en el siglo XIX, con la pretensión de terminar con la maldición que pesa sobre su mujer, Leonor Valdemar (Laia Marull), convertida en un espíritu tras un rito similar realizado cien años atrás, y de esta forma restituir la paz sobre sus antiguas posesiones.

Sin embargo, el rito, como ya había sucedido un siglo antes, termina con funestas consecuencias, y Cthulhu carga su ira sobre los invocadores. Solo Lázaro conseguirá su objetivo y salvará a su esposa, acabando con la maldición. Luisa, Nicolás y Ana sobrevivirán a la invocación, y mediante chantaje a los sectarios supervivientes, comprarán la libertad a cambio del silencio. La suprarrealidad quedará, nuevamente, soterrada, y las leyes naturales reinstituidas.

Ambas películas, como se ha comentado, supusieron el estreno como director de largometrajes de José Luis Alemán (1971), quien previamente había rodado 
algunos anuncios publicitarios, cortos como $H V$, mediometrajes como $H V 2$ y $H V_{3}$, y el reportaje de finalidad turística Isla de Pascua. De esa experiencia creó la productora La cruzada Entertaiment S. L. y elaboró el guión de estos dos filmes. Para su producción, consiguió en financiación propia trece millones de euros, un presupuesto digno de una superproducción. El propio Alemán declaró al respecto en la rueda de prensa previa al estreno de la primera de las películas que "mantenerse al margen de ayudas públicas responde a nuestro ideal de tener una total libertad a la hora de trabajar" (en Arce, 2010, Web).

A pesar de dichos elementos, los beneficios en taquilla no ayudaron a suplir los gastos de producción, pues el público español no respondió muy favorablemente y los intentos de distribución internacional resultaron infructuosos. Las críticas, en general, fueron negativas, aludiendo a la mediocridad de la cinta ${ }^{6}$. Lo mismo se puede apreciar de la visión que de esta producción ha quedado dentro del mundo académico, puesto que, siguiendo a Diego López y David Pizarro, La herencia Valdemar es...

... otro pequeño desastre cinematográfico escudado en Lovecraft, concretamente en El horror de Dunwich, donde un reparto insufriblemente inadecuado es suficiente para despedazar alguna que otra buena idea. Envuelta en desapariciones y antiguas mansiones de textura gótica, también jugando constantemente con el presente y el pasado a modo de anexión en la historia, el título se cristalizaba en trágico resultado (López \& Pizarro, 2013, pp. 409-410).

Esta mayoría de reacciones adversas llevaron al propio José Luis Alemán a escribir una carta abierta a los espectadores que fue publicada en Scifiworld el 25 de febrero de 2010. El director se defiende en este texto de las críticas vertidas en foros por parte del público, pidiendo perdón por haber dividido la película en dos partes -uno de los aspectos que explica las malas cifras de la película- y aludiendo que la división responde a razones de extensión del producto cinematográfico, y que, además, la sobreactuación de los actores se

\footnotetext{
6. Javier Ocaña en El País llegó a afirmar que "en demasiadas secuencias parece una película con medios profesionales comandada por un aficionado" (Ocaña, 2010, web). Por su parte, Edgar Egaña en Vavel, escribió que La herencia Valdemar "es todo un desperdicio: un presupuesto muy alto para el cine español, pero una evidente falta de talento cinematográfico" (Egaña, 2011: web). Pocas son las excepciones laudatorias, como la de Arce en La Butaca, quien indica que "El resultado es un bombón preciosista con el que el aficionado que sepa apreciar el conjunto global [...] gozará sobremanera más allá de obviedades y negativismos axiomáticos y puristas" (Arce, 2009, Web).
} 
realizó a propósito para buscar la esencia del cine de terror clásico de productoras como La Hammer, Universal o Tycon (Alemán, 2010b, Web).

\section{De Lovecraft a Alemán: fidelidades y desavenencias}

A la hora de analizar cuáles son las particularidades del díptico cinematográfico de Alemán y cuáles son los elementos lovecraftianos que en ella permanecen, hay que señalar, en primer lugar, que la interrupción de la trama entre ambas películas deja toda la resolución de los enigmas para la segunda parte, mediante un cliffhanger que no consiguió atrapar la atención del público. Ello se debe a que el final del primer filme cierra la historia enmarcada del matrimonio Valdemar, mientras que la trama principal únicamente ha sido presentada, por lo que no genera suspense en el espectador.

Por otra parte, respecto al modelo narrativo de la primera película, La herencia Valdemar comienza con unos veinte primeros minutos trepidantes, donde podemos ver a Luisa Llorente acudiendo a tasar la antigua mansión. Aquí aparecen todos los tópicos, en un acelerado ritmo narrativo, de la mansión encantada, con la presencia del monstruo al final: un asignación laboral imprevista, un primer tasador desaparecido, una foto con movimiento, un pueblo aislado fuera de cobertura, una casa abandonada, antigua y aislada, ruidos misteriosos, un fantasma captado por una cámara de fotos digital, puertas que se abren solas, el descubrimiento del cadáver en descomposición del tasador predecesor, Pablo Orquidia, y el necrófago que persigue a Luisa por las estancias de la casa, hasta que esta escapa. Sin embargo, es después secuestrada por el jardinero, Santiago (Santi Prego), y uno de los sectarios, Dámaso (José Luis Torrijo).

Entonces se desencadena la investigación, y con ella los antecedentes de la trama, es decir, el misterio de la mansión Valdemar, relatado por la Dr. Nora Cerviá (Ana Risueño) al detective Nicolás mientras viajan en un tren histórico hacia Montenegro. Es en este punto donde se desarrolla una extensa analepsis que ocupa el resto del primer filme, y que funciona como dilatado prólogo a la historia principal. La trama, entonces, se desarrolla en dos momentos narrativos, el ligeramente futurista (Terminal 5 de Barajas y vuelos en Zeppelín) y el del siglo XIX, que se unirán en el rito final donde se invoca a Cthulhu. Este 
largo flashback de La herencia Valdemar que desarrolla una tópica historia de amor romántica propia de una novela folletinesca, hacen al espectador olvidar la trama principal.

Aparece en esta historia secundaria, la desarrollada por el matrimonio Valdemar, otra de las fuentes aludidas de la película, la del folletín decimonónico. En ese sentido, la historia ambientada en el siglo XIX, de carácter sentimental, repite fórmulas y estereotipos en vez de buscar la originalidad. Así, entre otros rasgos, los personajes son propios de este género, siguiendo el estudio de Silvia N. Barei y Ana B. Ammann (1988, p. 38). El héroe (Lázaro Valdemar) es físicamente hermoso, es ingenuo e idealiza la realidad, predominan sus sentimientos sobre la razón y sufre injusticia e incomprensión del mundo que lo rodea; el oponente (Aleister Crowley) recibe los rasgos contrarios: físicamente desagradable, frío y calculador, acomodaticio, adepto a vicios y cobarde; la heroína (Leonor Valdemar) posee un papel inactivo, convertida en objeto, pues es el vínculo con lo sobrenatural.

Con la línea argumental ubicada en el diecinueve, José Luis Alemán se planteó realizar un proceso de ambientación. Para ello, se incluyeron acontecimientos muy puntuales del siglo XIX, como fueron el inicio del feminismo, la eclosión de la fotografía o el espiritismo. Sin embargo, se incurre en una actualización ideológica desde una perspectiva presente, con la idea de conectar con el público actual. El propio matrimonio está modernizado en su ideología, descrito durante el filme como adelantado a su tiempo. Además, se incluyen, aunque en un sentido anacrónico, personajes de la época. El más destacado es el ocultista, místico y alquímico Aleister Crowley (Paco Maestre), pero también debe citarse a los asistentes al primer rito de Dunwich: Lizzie Borden (Vanesa Suárez), Bram Stoker (Lino Braxe), y Belle Gunnes (Laura Toledo), todos ellos junto a un ficticio compositor de ópera italiano, Luliu Fuchini (Lucas Trapaza), que muere en el rito. Quizás de todas estas incorporaciones la más anacrónica sea la presencia del propio Lovecraft (Luis Zahera) en La sombra prohibida, teniendo en cuenta que en vida el escritor de Providence casi no viajó nunca y cuando lo hizo, jamás salió de Estados Unidos. Esta anacronía concreta responde a la idea apuntada al comienzo de que el escritor se ha convertido en elemento integrante de su propio mundo ficcional, esto es, ha sido absorbido por su propia creación. 
Conviene señalar en este punto que las modernizaciones son muy frecuentes en las adaptaciones fílmicas de la obra de Lovecraft. Entre estos cambios, lo más habitual es la adición de dos elementos inexistentes en la obra de H. P. Lovecraft: el sexo y el dinero. Muchas adaptaciones de Lovecraft incluyen el recurso del sexo, o al menos a la presentación de una relación amorosa, con la idea de enriquecer la trama de cara al público. No obstante, en este caso la historia de amor del matrimonio Valdemar resulta central, tanto que el segundo rito de Dunwich será el que resuelve el castigo que recayó al final de la primera película sobre ellos. Respecto al dinero, aparece en la trama a través de la investigación de Nicolás Trámel y su amigo Pablo Orquicia, el primer tasador, que observaron un desfalco dentro de Inmoberance y querían chantajear a la empresa en su propio beneficio, sin saber que dicho desfalco se destinaba a la secta y a ocultar las víctimas requeridas para la realización del rito de Dunwich.

Un elemento al que Lovecraft otorgaba gran relevancia a la hora de escribir un relato fantástico era la ambientación. En concreto, en El horror en la literatura afirmaba que "el factor más importante de todos es la atmósfera, ya que el criterio último de autenticidad no reside en que encaje la trama, sino que se haya sabido crear una determinada sensación” (Lovecraft, 1927, p. 11). Sin embargo, la creación de la atmósfera en las dos película de Alemán, aunque tiende hacia un progresivo oscurantismo según avanza la trama, se aleja de la concepción lovecraftiana. Ello se debe a que en muchos momentos los actores sobreactúan, dificultando empatizar con sus personajes, y los diálogos se vuelven superficiales, o se incluyen breves golpes de humor que rompen el clímax dramático del efecto fantástico.

Por otra parte, Lovecraft señalaba que en lo fantástico es necesario trabajar la credibilidad, y "esto solo puede conseguirse tratando el tema con cuidado realismo, excepto a la hora de abordar el hecho sobrenatural. Este hecho sobrenatural debe causar impresión y hay que poner gran cuidado en la construcción emocional; su aparición apenas debe sentirse, pero tiene que notarse" (Lovecraft, 1938, p. 175)7. No obstante, Alemán en sus películas no trabaja el realismo, pues la línea narrativa más contemporánea al lector incluye elementos que provocan alejamiento (Zeppelín, el tren histórico), y la otra línea

\footnotetext{
7. En cursiva en el original.
} 
narrativa se ubica en el siglo XIX, lo que supone un mayor alejamiento histórico. Con ello, el hecho sobrenatural queda más desligado de la realidad cotidiana del espectador, y se disminuye el impacto que genera su irrupción.

Conviene recordar en este punto que el género fantástico depende del efecto que causa en el espectador. Para que se produzca el efecto fantástico debe realizarse una identificación entre la realidad cotidiana de la obra y la del espectador, para que la irrupción de lo sobrenatural resulte sobrecogedora. La sensación de extrañamiento generada por Alemán no favorece a esa identificación, y el efecto fantástico se disipa, algo que sí tenía muy pendiente Lovecraft, con sus minuciosas descripciones de ambientes de Nueva Inglaterra.

Además, el proyecto de Alemán mira hacia un modelo clásico de terror. Si bien la propuesta puede funcionar a modo de homenaje, el director no atiende a una ley fundamental del género fantástico: la necesidad de que el espectador identifique el mismo código hermenéutico entre el universo narrativo y el suyo propio (Ferreras, 1995, p. 161). En lo fantástico hay que desmontar las defensas del espectador-personajes, sus concepciones racionales, y no hay mejor manera que atacar a las emociones (García Mingorance, 2014, p. 126), lo que obliga a actualizar constantemente el género, con la idea de buscar nuevas formas para comunicar el miedo.

Con este hecho, Alemán se aleja de las tendencias renovadoras del cine de terror español iniciadas en la década de los noventa y estacadas por Andre Willis: por un lado, la «ironía ecléctica», basada en la parodia cómica y el hibridismo de géneros para romper clichés y argumentos tradicionales, y cuyo claro ejemplo sería Acción mutante (1993), de Álex de la Iglesia; por otro, la de la «nueva sinceridad», preocupada por remarcar al máximo el realismo y la seriedad para hacer reflexionar al espectador, tendencia en la que cabe citar como ejemplo Los sin nombre (1999), de Jaume Balagueró (Willis, 2005).

Por ello, una vuelta al terror clásico, con cariz tópico, lleva a la película a desligarse del espectador modélico actual, lo que dificulta la identificación con la realidad ficcional: sobreactuación de los protagonistas, extrañamiento temporal, presencia de tópicos. Este aspecto se percibe si se compara con el 
modelo actual de fractura y fragmentación del cine fantástico ${ }^{8}$, alejado de ese gore y susto fácil. Ese cine fantástico actual se mueve más hacia procesos de ocultación de la información y la división de la historia en dos planos, uno de ellos oculto, que al revelarse al final desmontan las preconcepciones del espectador y le obligan a reconstruir toda la historia narrada, produciendo el efecto fantástico. Lo mismo sucede si se aleja del potencial impacto de la imagen para provocar el terror que ha ofrecido el punto de vista en primera persona tomado de diferentes videojuegos- y que tanto éxito ha cosechado en el cine actual, con ejemplos como [REC] (2007), de Jaume Balagueró y Paco Plaza.

Este alejamiento de las películas de Alemán respecto del modelo literario también se percibe en la génesis de los personajes. En La herencia Valdemar y en La sombra prohibida no se encuentran rasgos del héroe lovecraftiano, ese personaje con afán científico en busca de la verdad de forma meticulosa y que se topa con lo inexplicable, mostrando la dialéctica entre el materialismo científico y el deseo de trascendencia de la realidad que obsesionaba a Lovecraft. En el escritor de Providence, "sus personajes intentan aplicar el materialismo científico a realidades insospechadas, y generalmente salen muy mal parados si se acercan demasiado al entendimiento de esos fenómenos anormales” (González Grueso, 2013, p. 119).

El cambio realizado en los filmes de Alemán responde nuevamente a una modernización, con la idea de incluir personajes más cercanos al mundo del espectador actual, aunque ello elimine ese detalle del personaje racional enfrentado a los límites del racionalismo humano. Con ello, se produce una huida del fatalismo lovecraftiano, y del determinismo que marca la naturaleza de los personajes. Si en Lovecraft el protagonista, marcado muchas veces biológicamente, va en busca del misterio, investigando, con la idea de dotarlo de sentido, en La herencia Valdemar el misterio busca y domina a los personajes, que se ven involucrados de forma indirecta, por intermediación de otros: Aleister Crownley en el caso del matrimonio Valdemar, Maximilian Colvin en el

\footnotetext{
8. Se trata de un modelo de cine fantástico que juega con las pretensiones del espectador, manejando, mediante información oculta y solo aludida, una segunda trama que se revela al final, obligando a toda una interpretación de la película. Un ejemplo reconocible sería $E l$ sexto sentido (The Sixth Sense, 1999), de M. Night Shyamalan. Para una explicación más detallada de este modelo del cine fantástico actual, véase el estudio de Gabriel García Mingorance (2014, pp. 269-338).
} 
caso de Nicolás, Luisa, Ana y Eduardo. También destaca que en las películas la mansión Valdemar se yergue, al ser el núcleo de toda la acción, en verdadero protagonista, frente a los relatos lovecraftianos donde la carga dramática la lleva el narrador, junto a sus reflexiones sobre el hecho fantástico.

A ello hay que añadir el final feliz que busca la película, que nada tiene que ver con los términos de los relatos de Lovecraft, donde el encuentro con lo sobrenatural ha conducido a los protagonistas a la muerte, la locura, o les ha dejado marcados para el resto de su vida. A diferencia del maestro de Providence, cuyos relatos tienden hacia un desenlace trágico (disfórico), Alemán confiere a sus película un desenlace donde se termina positivamente con el drama: Maximilian muere en el rito; Lázaro consigue salvar a su mujer de la maldición; y Luisa, Ana y Óscar consiguen escapar con vida. Este final supone un dominio del bien sobre el mal, lo que plantea un sentido eufórico que satisface al público, pero que contradice el sentido de los textos de Lovecraft, que se caracterizan por la indiferencia de los seres sobrenaturales.

Sin embargo, quizás el mayor alejamiento respecto a la obra de Lovecraft operado en La herencia Valdemar y La sombra prohibida resida en que solo se tomen los elementos más reconocibles de los Mitos de Cthulhu, los que han configurado toda una cultura popular: la mención de la universidad de Miskatonic, la denominación del rito como la población de Dunwich, un ejemplar del Necronomicón, ese libro ficticio inserto en el universo lovecraftiano, que es estudiado en la película por Lázaro Valdemar; o la aparición del devorador en la primera película, y la presencia de Ctulhu al final del segundo filme. Muchas veces estos elementos aparecen como decorado en la película, carentes del sentido simbólico que poseen en los relatos de Lovecraft. El caso más determinante de este punto es el uso del Necronomicon como mera referencia -a modo de atrezo-, mientras que "Lovecraft utiliza el objeto libro como elemento catalizador de lo sobrenatural" (Ferreras, 1995, p. 67).

Por ello, resulta más complicado encontrar en el díptico de José Luis Alemán la esencia que caracteriza a los escritos de H. P. Lovecraft: la soledad del hombre ante la inmensidad del cosmos, la idea de una realidad superior ante la cual el ser humano se encuentra indefenso, o la incapacidad del hombre de aplicar conceptos científicos, ergo, racionales, a esa suprarrealidad. 


\section{Conclusiones}

Como se ha podido observar del análisis precedente, ante la dificultad de adaptar la obra del escritor de Providence al cine, José Luis Alemán, a la hora de realizar el díptico La herencia Valdemar, optó por una adaptación libre que toma un universo compartido y que se caracteriza por una divergencia estilística, y una ampliación del sentido del texto original al incluir otro tipo de fuentes. Sin embargo, dicha adaptación libre de los Mitos de Cthulhu incurre en diversos procesos de vulgarización antes explicados, como la simplificación (final feliz), la tendencia al maniqueísmo (el bien contra el mal), la actualización (el matrimonio Valdemar) y la modernización (ambientación retrofuturista).

Todo ello relega a Lovecraft a un conjunto de referencias de su universo ficcional más que a una lectura profunda de su obra. Por ello, ya señalaba el crítico Edgar Egaña: "La mala adaptación de los contenidos de H. P. Lovecraft salta a simple vista, ya que el novelista estadounidense escribía acerca de seres primigenios extraterrestres y no de demonios y espíritus, que es de lo que va la película de José Luis Alemán" (Egaña, 2011, Web).

A ello se añade que el díptico de Alemán acude a más fuentes a parte del universo ficcional del de los Mitos de Cthulhu: el cine clásico y el folletín. Por una parte, el modelo de terror clásico buscado por Alemán responde a los gustos de un espectador de lo fantástico impropio de la época actual. Por otra, en la película, cuya trama está basada, al modo del folletín, en la acumulación, hay una tendencia a explicar todos los detalles de la historia, sin legar interpretaciones al espectador ni dar espacio a la duda, cuando el efecto fantástico posee más fuerza si se basa más en la sugerencia.

Se percibe entonces que Alemán se aleja de la esencia de los textos de Lovecraft para tomar de ellos únicamente los aspectos que han conformado toda la cultura popular centrada en los Mitos de Cthulhu. Dichos elementos aparecen en este díptico cinematográfico configurando más una ambientación que adquiriendo el protagonismo y el poder de sugerencia que poseen en los escritos de H. P. Lovecraft. Debido a ello, se puede afirmar que Alemán no ha conseguido salvar el escollo que la mayoría de sus predecesores tampoco superó y la obra de Lovecraft reaparece nuevamente en el cine desde una lectura superficial de 
raigambre popular y dirigida a la masa de espectadores, y no desde la interpretación profunda de su obra literaria.

\section{Referencias Bibliográficas}

Aguilar, C. (2005). Introducción. En Carlos Aguilar (coord.) Cine fantástico y de terror español (1984-2004). Donostia-San Sebastián: Donostia Kultura, pp. 11-41.

Alemán, J. L. (2010a). La herencia Valdemar. España: La Cruzada Entertaiment, España.

Alemán, J. L. (2010b). Carta abierta del director José Luis Alemán sobre La Herencia Valdemar. En Scifiworld, 25 de febrero. Disponible en: http://www.elseptimoarte.net/carta-abierta-del-director-jose-luisaleman-sobre--la-herencia-valdemar--7570.html [26/09/2016].

Alemán, J. L. (2011). La herencia Valdemar II: La sombra prohibida. España: La Cruzada Entertaiment.

Alemán, J. L. [22/10/2015]. Nota del director. En La herencia Valdemar, Web oficial. Disponible en: http://www.laherenciavaldemar.com/ [26/09/2016].

Arce, J. (2009). La herencia Valdemar: El curioso caso de José Luis Alemán. En La Butaca.net, 24 de noviembre. Disponible en: http://www.labutaca.net/criticas/la-herencia-valdemar-el-curioso-casode-jose-luis-aleman/ [26/09/2016].

Arce, J. (2010). La cartelera se prepara para el reparto de La herencia Valdemar. En La Butaca.net, 20 de enero. Disponible en: http://www.labutaca.net/noticias/la-cartelera-se-prepara-para-el-repartode-la-herencia-valdemar/ [26/09/2016].

Arenas Orient, C. (2011). Lovecraft y la creación de atmósferas en el cine fantástico. En Ars Longa: cuadernos de arte, $\mathrm{n}^{\mathrm{O}}$ 20, pp. 231-241.

Barei, S. N., Ammann A. B. (1988). Literatura e industria cultural: del folletín al bestseller. Córdoba (Argentina): Alción editora.

Catalá, J. M. (2001). La puesta en imágenes. Conceptos de dirección cinematográfica. Barcelona: Paidós.

Egaña, E. (2011). La herencia Valdemar: fallida superproducción de terror española”, en Vavel, 15 de octubre. Disponible en: http://www.vavel.com/es/cine/12236-la-herencia-valdemar-fallidasuperproduccion-de-terror-espanola.html [26/09/2016].

Fernández Pinilla, S. (2008). La herencia Valdemar. El universo de Lovecraft. En Cameramán: Revista técnica cinematográfica, $\mathrm{n}^{0}$ 37, pp. 14-23.

Ferreras, D. (1995), Lo fantástico en la literatura y el cine: de Edgar A. Poe a Freddy Krueger. Madrid: Vosa.

García Mingorance, G. (2014). Fractura y fragmentación narrativa de lo extraordinario en cine y literatura. Alcalá: Fundación Universitaria Española. 
González Grueso, F. D. (2013). La ficción científica. Género, poética y sus relaciones con la literatura oral tradicional. El papel de H. P. Lovecraft como mediador. Madrid: Ediciones de la Universidad Autónoma de Madrid.

Gómez, T. (2002). Lovecraft, la antología. Barcelona: Océano.

Hernández de la Fuente, D. (2005). Lovecraft, una mitología. Madrid: ELR ediciones.

Houellebecq, M. (1991). H. P. Lovecraf: contra el mundo, contra la vida (trad. Encarna Castejón). Madrid: Siruela, 2006.

Jones, M. (2013). Tentacles and Teeth: The Lovecraftian Being in Popular Fiction”, en David Simmons (ed.), New Critical Essays on H. P. Lovecraft. New York: Palgrave Macmillan, pp. 227-247.

Llopis, R. (1969). Introducción. En H. P. Lovecraft y otros, Los Mitos de Cthulhu. Ed. Rafael Llopis. Trad. Francisco Torres Oliver. Madrid: Alianza editorial, pp. 11-44.

López, D., Pizarro, D. (2013). Silencios del pánico: Historia del cine fantástico y de terror español, 1897-2010. Barcelona: Tyrannosaurus Books.

Lovecraft, H. P. (1927). El horror en la literatura (Supernatural Horror in Literature). Trad. Francisco Torres Oliver. Madrid: Alianza, 1988.

Lovecraft, H. P. (1938). Notas sobre los escritos de literatura fantástica. En H. P. Lovecraft, La noche en el océano y otros escritos inéditos. Selección, preparación y prólogo de Alberto Santos Castillo. Madrid: Edaf, 1991, pp. 171-176.

Muñoz Casado. R. (2012). Los Mitos de Cthulhu como movimiento literario. Tesis Dirigida por Dr. Juan Manuel Nuñez Yusta. Madrid: Universidad Complutense de Madrid.

Ocaña, J. (2010). Película sin terminar. En El País, 22 de enero. Disponible en: http://elpais.com/diario/2010/o1/22/cine/1264114808_850215.html [26/09/2016].

Palacios, J. (2008). Lenguas muertas. Sobre «The Call of Cthulhu» de Andrew Leman. En Fernando Broncado y David Hernández de la Fuente (ed.), Cuadernos del abismo. Homenaje a H. P. Lovecraft. Madrid: Literaturas Comunicación, pp. 197-199.

Sánchez Noriega, J. L. (2000). De la literatura al cine: teoría y análisis de la adaptación. Barcelona, Buenos Aires, México: Paidós.

Sharrett, C. (2015). The Haunter of the Dark: H. P. Lovecraft and Modern Horror Cinema. En Cineaste: America's Leading Magazine on the Art and Politics of the Cinema, vol. 41, $\mathrm{n}^{\mathrm{0}}$ 1, pp. 22-26.

Smith, D. G. (2005). H. P. Lovecraft in Popular Culture: The works and Their Adaptations in Film, Television, Comics, Music and Games. Jefferson (North Carolina): McFarland \& Company.

Willis, A. (2005). From the margins to the mainstream: trends in recent Spanish horror cinema. En Antonio Lázaro Reboll y Andrew Willis (ed.), Spanish Popular Cinema. Manchester: Manchester University Press, pp. 237-249. 
Woodward, F. H. (2008). Lovecraft: Fear of the Unknown. Estados Unidos: Wyrd Studios.

\section{Equipo técnico de La herencia Valdemar y La sombra prohibida}

Director: José Luis Alemán.

Productor ejecutivo: Iñigo Marco.

Director de producción: Miguel Ángel González.

Director de arte: Luis Valles (Koldo).

Director de fotografía: David Alzano.

Figurinista: Bina Diageler.

Jefe de sonido: Antonio Rodríguez (Mármol).

Maquillaje: Caitlin Acheson.

Peluquería: Mara Collazo.

Montador: Frank Gutiérrez.

Músico: Arnau Bataller.

Efectos especiales: Reyes Abades.

Cómo citar: Peregrina Castaños, M. (2017). De Lovecraft a Alemán: una adaptación cinematográfica de los Mitos de Cthlhu. Fotocinema. Revista científica de cine $y$ fotografía, $\mathrm{n}^{\mathrm{o}}$ 14, pp. 233-253. Disponible: http://www.revistafotocinema.com/ 